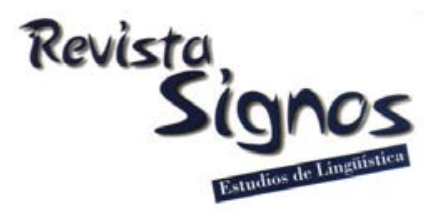

\title{
Representaciones mentales en la comprensión del discurso: Del significante lineal al modelo de situación
}

\author{
Mental representations in discourse comprehension: \\ From lineal signifier to situation model
}

\author{
Bernardo Riffo \\ UNIVERSIDAD DE CONCEPCIÓN \\ CHILE \\ bernardo.riffo@udec.cl
}

Recibido: 04-XII-2015 / Aceptado: 25-V-2016

\begin{abstract}
Resumen
En este artículo se presentan y discuten diversos niveles de representaciones mentales y los mecanismos implicados en su elaboración durante el proceso de comprensión del discurso. Fundamentalmente se distingue entre aquellas representaciones de carácter estrictamente lingüístico, asociadas a la estructura lineal del significante y las vinculadas al significado y los contenidos, cuya naturaleza no presenta un carácter lineal. Tal distinción, que se encuentran presente en la Lingüística desde sus orígenes, se mantiene en los modelos psicolingüísticos actuales. Dentro de las representaciones aquí tratadas, se profundiza la discusión en torno a la de modelo de situación, se da cuenta de sus fundamentos y se la contrasta con nuevas perspectivas como las teorías corpóreas.
\end{abstract}

Palabras Clave: Modelo de situación, comprensión del discurso, representaciones mentales.

\begin{abstract}
This article presents and discusses several levels of mental representations and the mechanisms involved in their development during the process of discourse comprehension. Fundamentally, it distinguishes those representations strictly linguistic, associated with the linear structure of language, from those linked to the meaning and content, whose nature has a not linear character. These distinctions, which are present in linguistics from its origins, remain at current psycholinguistic models. Among the mental representations here presented, we discuss deeper around the concept of situation model, analyzing its foundations and contrasting it with new perspectives, such as the embodiment theories.
\end{abstract}

Key Words: Situation model, discourse comprehension, metal representations. 


\section{INTRODUCCIÓN}

En el presente artículo buscamos dar cuenta del complejo proceso que subyace a la comprensión del discurso, centrando la atención en aquellos mecanismos y representaciones que hacen posible que, desde una señal lingüística de carácter linealsecuencial, nuestro cerebro logre elaborar una representación no lineal, análoga y modal, una réplica cognitiva de la situación referida por el texto. Esta explicación psicológica del lenguaje tiene antecedentes que se remontan a los inicios de la lingüística moderna. En efecto, la comprensión del lenguaje constituye un proceso mediante el cual, a partir de un mensaje físicamente sustentado, el receptor, haciendo uso de su conocimiento lingüístico y otros recursos cognitivos, elabora en la memoria una representación del contenido de dicho mensaje. No nos situamos aquí en el plano del sistema lingüístico, sus niveles de organización y las unidades que los integran, sino en el de las operaciones que los hablantes realizan con el lenguaje. La comprensión es, por tanto, un hecho de 'habla' en términos saussurianos (o de 'actuación' para la teoría generativa) y, en cuanto tal, implica elementos físicos, psicológicos (perceptivos, representacionales) y fisiológicos, comunicativa y socioculturalmente enmarcados. En este contexto, el presente artículo se propone presentar tales mecanismos para, a la postre, discutir la naturaleza y funciones del llamado modelo de situación según el estado actual de la investigación.

\section{Un marco psicolingüístico}

\subsection{Carácter psicológico de las representaciones del lenguaje}

La perspectiva psicolingüística desde la cual nos proponemos dar cuenta del fenómeno supone la existencia de ciertas formas de representación mental como unidades constitutivas del lenguaje. Dicho supuesto no es original de la psicología cognitiva actual; ya en los inicios de la lingüística, Saussure (1916) postulaba el carácter psicológico de las representaciones del lenguaje, donde el signo lingüístico constituye una 'entidad psíquica'1 de dos caras. Por una parte, se postula una representación de carácter lineal correspondiente a la forma fonológica de la palabra -'significante'- y, por otra, un concepto asociado a ella -'significado'-, de carácter no lineal. Cabe enfatizar en este punto que ambas partes del signo lingüístico tienen un carácter psíquico y, como tales, deben ser consideradas representaciones mentales. Así, la forma fonológica de la palabra no corresponde a los sonidos (cadena fónica) ${ }^{2}$ que la conforman, sino a su huella sensorial en la memoria. El concepto, a su vez, presenta una configuración distinta, pudiendo ser definido como un conjunto de elementos que constituyen propiedades de una entidad o evento en términos de rasgos o categorías.

En todo sistema lingüístico las unidades léxicas, que ya están constituidas por unidades subléxicas (fonemas, sílabas, morfemas), se relacionan con otras conformando, de acuerdo con ciertos principios sintácticos, secuencias de palabras que integran unidades mayores llamadas sintagmas y oraciones. De manera 
equivalente a lo que sucede en el nivel léxico, encontramos en el nivel oracional una distinción básica dual en términos de su representación en la memoria, a saber, una correspondiente a la 'forma sintáctica' de la oración y otra al significado asociado a esta en la forma de una 'proposición'. Esto tiene importantes consecuencias psicolingüísticas para la comprensión, toda vez que el procesamiento implica la elaboración de ambos tipos de representación.

La unidad estructural llamada oración, sin embargo, no constituye el límite último de la organización del mensaje; en efecto, estas oraciones pueden formar otros encadenamientos mayores, dando lugar a textos o discursos ${ }^{3}$. Así, los mensajes que intercambian los miembros de la comunidad lingüística casi siempre superan el límite de la oración. Con todo, se distingue también aquí entre la representación de la forma lingüística, por una parte, y el significado asociado a ella, por otra, es decir, entre la huella psicológica de la secuencia de oraciones (en términos fonológicos y sintácticos) y su correspondiente semántica, una red proposicional jerárquicamente organizada. Esta dualidad representacional se vuelve a su vez más compleja al incluir una tercera posibilidad, a saber, el 'modelo de situación' (van Dijk \& Kintsch, 1983; Kintsch, 1988, 1998), noción que desarrollaremos en detalle en la sección 2. Antes de ello, y a objeto de establecer bases psicolingüísticas pertinentes, nos referiremos a los mecanismos de comprensión implicados en la elaboración de las diversas representaciones tratadas en este artículo.

\subsection{La comprensión del lenguaje en el circuito del habla}

En el circuito de la comunicación, la recepción del mensaje (el texto, para los intereses del presente artículo) sigue un camino inverso al de su producción, involucrando los mismos componentes de carácter físico, fisiológico y psicológico que hicieron posible su elaboración. Si bien hoy disponemos de un conocimiento más preciso que nos permite explicar con meridiana claridad el papel que cada uno de estos elementos desempeña en el proceso e identificar las distintas fases de su curso temporal, también en esta materia el Curso de Lingüística General ofrece propuestas que, en su momento, anticiparon algunos desarrollos más recientes de la psicolingüística y las neurociencias. Entre los elementos implicados se encuentran: 1) un plano físico, que constituye el soporte material del texto (acústico o visual si se trata de texto oral o escrito ${ }^{4}$ ); 2) un plano fisiológico, en el que se sitúa la percepción del estímulo auditivo o visual y que permite el traspaso de las señales acústicas o luminosas hacia el cerebro; y 3) un plano psicológico, donde el mensaje se reconstruye mentalmente en sus representaciones fundamentales de significante y significado.

Un siglo después de Saussure, la psicolingüística actual, disciplina con un marcado carácter empírico, ha desarrollado métodos conductuales (medidas cronométricas, registro de movimientos oculares, etc.) y neurofisiológicos (ERP, fMRI, PET), e incorporado técnicas de estadística avanzada (análisis multinivel, modelos bayesianos, 
ecuaciones estructurales, entre otros), al mismo tiempo que ha elaborado complejos modelos teóricos que hacen posible una explicación más precisa de la comprensión del lenguaje, abriendo con ello una ventana a la mente humana (Speer, Reynolds, Swallow \& Zacks, 2009; Ratcliff, 2012; Rayner \& Kliegl, 2012; Pulvermüller, 2013).

\subsection{Memoria y comprensión}

Un papel preponderante en el procesamiento del lenguaje le cabe a la memoria, entendida como un complejo sistema responsable de la percepción, codificación, almacenamiento y recuperación de representaciones mentales en diversos formatos y dimensiones. Desde los inicios de la psicología se ha postulado - con el respaldo de abundante, consistente y robusta evidencia empírica- el carácter no unitario de la memoria mediante modelos teóricos que proponen la existencia de distintos componentes mnemónicos. Una de las propuestas más reconocidas es el llamado modelo modal (Atkinson \& Shiffrin, 1968), que distingue tres espacios, a saber, 1) la memoria sensorial, responsable de convertir las propiedades estimulares de la señal en una representación interna pre-categorial, materia prima para la elaboración de estructuras más complejas; 2) la memoria de corto plazo (MCP), encargada de realizar operaciones de codificación categorial; y 3) la memoria de largo plazo (MLP), que recibe los productos elaborados en la memoria de corto plazo almacenándolos por periodos extensos de tiempo, considerada depositaria del conocimiento de un individuo.

En la comprensión del discurso, la memoria sensorial desempeña un papel elemental pero imprescindible transformando los rasgos visuales (en el caso del texto escrito) en representaciones internas en la forma de unidades icónicas o las propiedades acústicas (en el caso del texto oral) en representaciones ecoicas ${ }^{5}$, operación fundamental para el procesamiento del lenguaje al permitir la formación de las representaciones básicas necesarias para acceder al significado. La memoria de corto plazo, a su vez, ejecuta las tareas implicadas en el procesamiento de la información proveniente de la memoria sensorial, cuyas representaciones reciben un tratamiento para ser categorizadas según formatos (verbal, visual, etc.). Debido a estas funciones, la MCP ocupa un lugar clave en el procesamiento del lenguaje realizando operaciones de cómputo que implican la elaboración de representaciones mentales para lo cual debe, además de utilizar los productos provenientes de la memoria sensorial, recuperar información de la MLP. Por lo mismo, Baddeley (1986) propuso el concepto de memoria de trabajo ${ }^{6}$ (working memory) agregando un componente ejecutivo central y más tarde un almacén episódico (Baddeley, 2000), dispositivos que permiten a este elemento del sistema mnemónico desempeñar simultáneamente las tareas de procesamiento y retención de la información. La MLP, que almacena representaciones organizadas categorialmente, dispone de información cristalizada de persistencia mayor; forman parte de ella el lenguaje, el conocimiento del mundo y el saber operar para las distintas tareas que el sistema cognitivo debe resolver. Una 
importe distinción en este espacio es la que diferencia entre un componente referido a representaciones inconscientes (memoria implícita) y otro explícito (memoria declarativa), en el que, a su vez, es posible diferenciar representaciones generales en forma de esquemas, marcos y competencias (memoria semántica o socio-cultural), y otro dedicado a almacenar información específica de experiencias individuales (memoria episódica) (Tulving, 1972).

Abundante evidencia tanto conductual como neurológica respalda las distinciones antes señaladas. En efecto, hallazgos de investigación experimental y datos clínicos confirman que diferentes estructuras y mecanismos cerebrales participan en tareas de recuerdo cuando estas implican representaciones generales descontextualizadas, a diferencia de aquellas que incluyen evocar experiencias individuales situadas en un contexto. Del mismo modo, estas distinciones concuerdan con la propuesta de dos mecanismos neurales diferenciados en el cerebro: uno para la actividad eléctrica efectiva en un momento determinado y otro para la conexión estable entre las neuronas (Squire, 1987; Baddeley, 2000; Hart \& Kraut, 2007).

Como dijimos al comienzo, la comprensión del lenguaje supone la decodificación de la cadena lineal del significante para elaborar una representación no lineal del contenido del mensaje. Dicho proceso involucra todos los niveles del sistema lingüístico, desde el fonema hasta el discurso. Complejos procesos y mecanismos hacen posible la percepción del habla, que resuelve grandes dificultades que presenta la inestabilidad de la señal (el problema de la ausencia de invariantes), convirtiendo las propiedades acústicas en representaciones fonológicas (en el caso del texto oral), lo mismo que con las señales gráficas (letras) en unidades discretas (Marslen-Wilson, 1987; Liberman \& Mattingly, 1989). Factores como la frecuencia, lexicalidad, y longitud de las palabras han mostrado gran poder predictor en los procesos de reconocimiento de palabras. Las representaciones producto de estos procesos constituyen unidades de la mente asociadas a significado en un nivel léxico.

Los encadenamientos de palabras (oraciones), a su vez, se procesan mediante un mecanismo incremental que incluye varias fases, a saber, 1) la segmentación de la cadena o parsing, 2) la asignación de papeles estructurales a estos segmentos o constituyentes, 3) el establecimiento de dependencias o relaciones entre los constituyentes, 4) el acoplamiento sintáctico semántico, y finalmente 5) la construcción de una representación proposicional de la oración (Belinchón, Igoa \& Rivière, 2005). Este último paso es de gran interés para el presente artículo, puesto que la proposición, en tanto representación del significado asociado a la oración, constituye un producto decisivo para explicar la comprensión del discurso, como veremos en las secciones que siguen. 


\section{Representaciones del discurso}

Como vimos en los apartados anteriores, tanto la lingüística moderna en sus orígenes, como posteriormente la psicolingüística han discernido entre las representaciones de la forma y del contenido tratándolas como objetos distintos. Los estudios de comprensión del discurso de las últimas décadas, a su vez, con enfoques predominantemente cognitivos, han incorporado una tercera representación en sus modelos teóricos. Así, desde van Dijk y Kintsch (1983), se reconocen tres representaciones, a saber, 1) la de superficie, 2) la base textual y 3) el modelo de situación (Kintsch, 1988, 1998; McNamara \& Magliano, 2009; Gernsbacher \& Kashak, 2013).

\subsection{La representación de superficie}

La primera de ellas corresponde a una representación literal de la forma lingüística (ortográfico-fonológica, sintáctica) del texto. En otras palabras, se trata de una amalgama compuesta por la forma fonológica de las palabras (y su expresión escrita alfabética ${ }^{7}$ ), por una parte, y la forma sintáctica de las oraciones que constituyen el texto, por otra. En términos psicológicos, en cuanto su persistencia temporal, la representación de superficie es efímera, tanto que desparece rápidamente en el lapso de tiempo que las representaciones se encuentran activas en la memoria de trabajo, perdiéndose luego y siendo muy difícil recuperarlas más allá de los límites temporales de la memoria operativa (Gernsbacher, 1985; Kintsch, Welsch, Schmalhofer \& Zimny, 1990). A medida que el lector avanza en el texto, la representación se va haciendo cada vez más inaccesible, tanto así que en una tarea de evocación el desempeño es muy pobre o nulo. Respecto de la información que contiene esta representación, se reconoce que es de carácter perceptivo y está referida a la forma del texto (fundamentalmente ortográfico-fonológica a partir de texto oral o escrito, y sintáctica, basada en el conocimiento de la lengua y en las representaciones gramaticales implicadas en el procesamiento del lenguaje, como se adelantó en apartados anteriores).

\subsection{La base textual}

La base textual, por su parte, que contiene información semántica explícita en el texto, se diferencia de la representación de superficie tanto en la naturaleza de su contenido, como en su persistencia temporal en la memoria. Integrada por el conjunto de proposiciones derivadas de la secuencia de oraciones del texto, la base textual constituye una estructura jerárquica organizada en niveles de micro y macroestructura cuya configuración responde, entre otros, a ciertos principios de formación textual que incluyen la diversidad discursiva de la comunidad lingüística ${ }^{8}$. Debido a que el texto contiene abundante información implícita - de hecho, la información explícita en la superficie textual constituye solo una pequeña porción de todo lo que se requiere para comprender el texto-, las proposiciones que integran la base de texto representan 
los contenidos semánticos explícitos y, en consecuencia, presentan numerosos vacíos que el lector debe completar haciendo uso de su conocimiento del lenguaje y, sobre todo, de su conocimiento del mundo disponible en la memoria declarativa de tipo semántico, como señalamos antes. Bajo tales circunstancias, las representaciones proposicionales que conforman la base de texto no permiten realizar todas las operaciones de llenado de vacíos necesarias para elaborar una representación coherente del texto -requisito fundamental de la comprensión-; algunos elementos de ella, sin embargo, hacen posible lograr ciertos vínculos que unen algunas piezas básicas para operaciones de asignación de coherencia. Ello, sin embargo, dista mucho de ser suficiente para una comprensión cabal del texto.

No obstante su origen en la lógica, donde se propusieron como herramientas inferenciales 'despsicologizadas' (Frege, 1918) ${ }^{9}$, las proposiciones fueron incorporadas más tarde por la psicología cognitiva de corte simbólico-computacional como representaciones mentales del significado conceptual asociado a expresiones lingüísticas, específicamente, a las oraciones. Dos supuestos fundamentales subyacen a la noción de proposición, a saber, 1) que la mente es una máquina simbólica que computa unidades de representación utilizando una suerte de lenguaje interno -como el mentalés de Fodor (1983) - para realizar las operaciones de cómputo, y que 2) los significados son 'amodales', es decir, tienen un carácter abstracto e independiente de las propiedades perceptivas de las entidades que denotan. Aunque en la literatura se reconocen diversas propuestas (Anderson \& Bower, 1983), ha sido el modelo proposicional de Kintsch el que mayor desarrollo e influencia ha alcanzado (Kintsch, 1974, 1988, 1998; van Dijk \& Kintsch, 1983). De importancia central para esta propuesta es el valor de las proposiciones como unidades de representación que permiten explicar la conducta; así, abundante evidencia empírica de la realidad psicológica de las proposiciones se encuentra en diversos estudios que dan cuenta de ello mediante experimentos en los que se ha medido el recuerdo (guiado mediante claves o libre), el efecto priming o el tiempo de lectura (Anderson \& Bower, 1973; Kintsch \& Keenan, 1973; Kintsch, 1974; Ratcliff \& McKoon, 1978; Graesser, 1981; Haberlandt \& Graesser, 1985; de Vega, Carreiras, Gutiérrez-Calvo \& Alonso-Quecuty, 1990). Aun con todo el poder explicativo otorgado a las proposiciones, cabe cuestionar aquí qué valor funcional tienen para el procesamiento y la comprensión, toda vez que ellas representan los contenidos explícitos, como lo considera el modelo de Kintsch (1974, 1988, 1998).

\subsubsection{Inferencias y base de texto}

En este punto del proceso, las inferencias desempeñan un papel central al unir las piezas de información estableciendo vínculos entre ellas; por ello, no es extraño que constituyan un nudo crítico en los modelos teóricos (van Dijk \& Kintsch, 1983; Graesser, 1981; Gernsabcher, 1990; Kintsch, 1998). Fundamentalmente, el papel de 
estas operaciones es agregar la información 'faltante', aquello que el texto no expresa en la superficie. Por lo mismo, los productos que se originan por medio de las inferencias no forman parte de la representación de superficie; pero tampoco -stricto sensu- de la base de texto. En un intento por resumir su función, Vieiro y Gómez (2004) señalan que las inferencias participan de todo el proceso de comprensión y se generan a partir de diferentes fuentes como a) el conocimiento general, b) el conocimiento de dominios específicos, y c) representaciones específicas basadas en experiencias particulares a partir de lecturas previas o del propio texto en proceso. La controversia, no obstante y como sucede habitualmente en las ciencias, no ha estado ausente en este tema; así, mientras algunos modelos proponen una abundancia de inferencias durante la lectura (incluso más de las necesarias para la coherencia), otras sostienen una posición minimalista restringiéndolas solo a aquellas estrictamente requeridas para el proceso en tiempo real, en tanto que las inferencias de elaboración más compleja se harán necesarias en la media que la tarea de comprensión lo requiera de manera particular (McKoon \& Ratcliff, 1992). En el modelo de construcciónintegración, Kintsch (1998) distingue entre aquellas inferencias automáticas -que incluyen inferencias puente u otras llamadas 'transitivas' en un dominio familiar- y las controladas que requieren procesos estratégicos -que incluyen la búsqueda de conocimiento para realizar operaciones de unión entre piezas del discurso y las inferencias lógicas implicadas en la resolución de problemas (Kintsch, 1993, 1998). Con todo, está fuera de cualquier duda que los procesos inferenciales responden a la necesidad de llenar los vacíos de información que la base textual presenta, lo que solo es posible mediante otras formas de representación de una naturaleza distinta a la lingüístico-conceptual de las proposiciones. Como el mismo Kintsch lo propone, los procesos inferenciales tienen lugar durante la construcción del modelo de situación (Kintsch, 1993, 1998).

\subsection{El modelo mental en la comprensión del discurso}

La tercera de las representaciones elaboradas durante la comprensión, que ha recibido el nombre de escenario, modelo del discurso, modelo mental o modelo de situación ${ }^{10}$, consiste en una suerte de amalgama entre la información explícita en el texto (base textual) y el conocimiento de mundo del comprendedor (Gernsbacher \& Kaschak, 2013). Aunque guarda importantes coincidencias con la noción de JohnsonLaird (1983), particularmente en la tradición psicolingüística de los estudios del discurso, ha prevalecido la última de estas denominaciones, vinculada originalmente al modelo estratégico (van Dijk \& Kintsch, 1983) y luego al modelo de construcciónintegración (Kintsch, 1988, 1998). Ambas propuestas conciben dicha representación como un objeto de naturaleza no verbal e incluso no semántico-conceptual (en el sentido lingüístico). A diferencia del carácter amodal que define a las proposiciones, se trata de un constructo cognitivo sobre aquello que el texto trata e implica la elaboración de una 'simulación mental' compleja que incluye, entre otras, propiedades 
de naturaleza viso-espacial, acústica y, en principio, de origen diverso (las que podrían corresponder cualquiera de las modalidades de los sentidos, según veremos en adelante, dependiendo de la propuesta teórica que se considere).

\subsubsection{Dimensiones del modelo}

Zwaan y colaboradores (Zwaan, Langston \& Graesser, 1995; Zwaan \& Radvansky, 1998) propusieron, en función de la comprensión de textos narrativos en el marco del 'modelo de indexación de eventos', cinco dimensiones que caracterizan el modelo de situación, a saber: a) tiempo, b) espacio, c) protagonista, d) intencionalidad, y e) causalidad. El comprendedor indexa los eventos en un marco temporal de la historia; esta, a su vez, ocurre situada en un cierto espacio o región; la historia involucra a un(os) protagonista(as); unos eventos vinculados causalmente entre sí; los eventos, además, se vinculan con los objetivos del(os) protagonista(s). De estas especificaciones incluidas en la propuesta de Zwaan y su equipo (Zwaan et al., 1995; Zwaan \& Radvansky, 1998) se derivan, entre otras implicancias, que la integración de nuevos elementos durante la comprensión dependerá del estado del modelo de situación y la relación que con este tenga la información entrante; del mismo modo, mientras un mayor número de dimensiones se encuentren interrelacionadas con un evento, mayor será la probabilidad de su integración exitosa y menor el consumo de recursos que la tarea demande (v. gr., tiempo).

Numerosos estudios han proporcionado evidencia de que los comprendedores construyen un modelo de situación en tiempo real (on-line) con las propiedades arriba señaladas. Es esta representación la que provee el marco o escenario que posibilita la elaboración de inferencias y contribuye, al mismo tiempo, a explicar porqué es posible la realización de dichas operaciones (Radvansky, 2009).

De las cinco dimensiones propuestas por Zwaan y colaboradores (Zwaan et al., 1995; Zwaan \& Radvansky, 1998), espacio y tiempo -en este orden- han recibido mayor atención, siendo escasos, en cambio, los estudios en los que todas ellas sean consideradas en su conjunto (Therriault, Rinck \& Zwaan, 2006). Varios trabajos de tipo experimental con técnicas conductuales proporcionan evidencia a favor de la hipótesis de que los lectores elaboran una representación del lugar (un recinto, por ejemplo) en el que ocurren los eventos de una historia o descripción. Así, en función del punto en el que se ubica un objeto o personaje dentro del escenario que se presenta en el texto, la información textual puede ser incorporada con mayor o menor facilidad (en términos del consumo de tiempo, la principal medida utilizada en este tipo de estudios), lo que constituiría una prueba de que el procesamiento del discurso conlleva la construcción de una representación espacial análoga. Tal conclusión, sin embargo, ha sido objeto de discusión y no existen datos concluyentes; tanto así que algunos autores postulan que una representación espacial análoga, como parte del modelo de situación, solo ocurre cuando el texto es explícitamente espacial o cuando 
hay una razón estratégica para ello (Langston, Krammer \& Glenberg, 1998). El tiempo, a su vez, como una dimensión del modelo mental, ha sido objeto de investigación en varios estudios, cuyos resultados demuestran que los lectores construyen una representación temporal de los acontecimientos en una historia; de esta forma, se ha encontrado que la distancia entre dos eventos en una línea temporal se refleja en la velocidad de procesamiento, más lenta para procesar un fragmento del texto con mayor desplazamiento temporal con respecto a un punto anterior en la historia (Zwaan, 1996).

\subsubsection{Naturaleza, propiedades y funciones del modelo en tiempo real}

Como hemos visto hasta aquí, el modelo de situación constituye una representación modal, no lineal, análoga y multidimensional que implica realizar un 'simulacro mental' de la situación referida por el texto, acto que, bajo ciertas condiciones, puede considerarse una suerte de 'experiencia vicaria' para el lector. Numerosa y consistente evidencia empírica respalda la hipótesis de que esta representación se elabora desde el comienzo del proceso de comprensión, desplegándose tempranamente como un marco para la integración de la información que las sucesivas oraciones (y sus proposiciones derivadas) van aportando. De este modo, la construcción del modelo se desarrolla mediante un proceso dinámico que incluye la elaboración y modificación de este 'escenario mental' en el curso de la lectura y permite dar cuenta de la perspectiva que adopta el lector (Sanford \& Garrod, 1981; Garrod \& Sanford, 1983). La representación del escenario permite la mantención de elementos activos tanto en el foco como en el trasfondo y es otra dimensión en la que se aprecia el papel del modelo en la dinámica de la lectura (Glenberg, Meyer \& Linden, 1987). En la misma línea, otras investigaciones han aportado evidencia de que el modelo se elabora desde la perspectiva del lector, reflejando una suerte de 'ojo mental' que observa la escena representada, reforzando la noción de experiencia vicaria del lector como una suerte de observador participante de la escena (Sanford \& Garrod, 1981; Garrod \& Sanford, 1983; Radvansky, 2009). En esta dinámica, el modelo mental, escenario o modelo de situación, en tanto representación no lingüística y no proposicional del discurso, no solo interviene tempranamente en el procesamiento, sino que, junto con proporcionar las condiciones que hacen posible la realización de inferencias para la asignación de coherencia (y la consiguiente comprensión del discurso), se actualiza en tiempo real a medida que se desarrolla el proceso de lectura (Sanford \& Garrod, 1981; Garrod \& Sanford, 1983; Radvasky \& Dijkstra, 2007; Radvasky, 2009).

Aun con todas las ventajas arriba presentadas, los modelos o escenarios no han estado exentos de crítica, las que apuntan principalmente a limitaciones derivadas de su falta de precisión y especificación formal, ya que no está claro cuál es el código de su elaboración ni los primitivos representacionales que los constituyen (de Vega et al., 1990). Entre otras consecuencias de esto, encontramos que no siempre resulta posible 
distinguir con adecuada nitidez entre los 'esquemas' o 'marcos' (Rumelhart, 1980) y los modelos (mentales o de situación), aunque sabemos que los primeros corresponden a prototipos abstractos almacenados en la memoria semántica, mientras que los últimos se elaboran ad hoc en la memoria episódica como parte de la experiencia individual del lector con el discurso.

No obstante la existencia de estas distinciones, sobre las que se tiene un amplio consenso así como respecto de la necesidad de los modelos -o una forma de representación equivalente- para explicar cabalmente la comprensión, cabe preguntarse por la naturaleza de estos objetos y su lugar en la cognición humana. Si además, como se ha postulado con gran acuerdo entre los autores, los modelos integran información proveniente tanto del conocimiento del lenguaje disponible para el lector, como de su conocimiento del mundo, no existe el mismo grado de consenso sobre cómo se constituyen los modelos ni cuáles son las múltiples fuentes de las cuales se obtienen los elementos para su elaboración episódica. En el actual estado del arte, dos aproximaciones postulan distinta naturaleza para los modelos. Por un lado, es posible concebirlos según el molde de las teorías proposicionalistas como la de Kintsch (1974, 1988, 1993, 1998; van Dijk \& Kintsch, 1983), las que sostienen que para la elaboración del modelo se emplean categorías similares a las de las proposiciones, vale decir, predicados y argumentos (ACCIÓN, AGENTE, OBJETO, INSTRUMENTO, etc.). Más allá del valor intrínseco de las proposiciones como unidades de representación conceptual abstracta y amodal asociadas a una expresión sintáctica en el texto, esta perspectiva postula que el conocimiento general puede organizarse en una gran red proposicional. De este modo, en el modelo de construcción-integración (Kintsch, 1988, 1998), las representaciones proposicionales proveen un poderoso mecanismo para determinar qué conocimiento se encuentra disponible para el lector y cómo se pueden realizar las inferencias necesarias para, desde la base proposicional, elaborar el modelo de situación. Las proposiciones, entonces, constituyen el medio representacional para la elaboración del modelo.

Por otro lado, en forma alternativa en las últimas décadas se han desarrollado propuestas teóricas respaldadas por abundante evidencia empírica tanto de corte conductual como neurofisiológico que sostienen una concepción de los procesos cognitivos 'corporeizada', donde el mecanismo de representación para un modelo no es proposicional, sino más bien análogo. Un elemento central en estas propuestas es la idea de que las representaciones semánticas elaboradas a partir de expresiones verbales incluyen componentes que conservan la(s) modalidad(es) sensorio-motora(s) involucrada(s). Dicho postulado, que constituye un punto crítico de este enfoque, contrasta radicalmente con el carácter amodal de las proposiciones; en efecto, mientras estas prescinden de cualquier rasgo perceptivo o motor asociado a las situaciones denotadas, las representaciones corpóreas incluyen, en su génesis, los rasgos que las vinculan modalmente con aquello que representan (Barsalou, 1999; 
Zwaan, 2003, 2015; de Vega, 2005; de Vega, Glenberg \& Graesser, 2008). La comprensión del lenguaje constituye, desde esta perspectiva, una experiencia indirecta provocada por tres procesos: activación, conceptualización e integración de representaciones que, básicamente, se encuentran vinculadas a los mecanismos de percepción y control motor (Zwaan, 2003). La evidente discrepancia que esta perspectiva muestra con respecto a las propuestas amodales ha situado el debate en el centro de la discusión sobre la naturaleza de las representaciones mentales y su papel en los procesos cognitivos, en general, y en la comprensión del lenguaje (y del discurso), en particular. Aunque las propuestas corpóreas, por su origen, han resultado más adecuadas para dar cuenta de aspectos más concretos de las representaciones (sobre todo aquellos relacionados con la acción, la percepción y el espacio), también han mostrado cierta capacidad para explicar la representación de significados abstractos, muchas veces por la vía de una metáfora corpórea, la que proporciona el anclaje al plano sensorio-motriz (Zwaan, 2015). Cabe preguntarse, sin embargo, cuál es su alcance para dar cuenta de procesos inferenciales más complejos y cuáles primitivos representacionales o unidades mínimas constituyen el código fundamental de representación. Del mismo modo, resulta pertinente la cuestión acerca del grado de formalización que dicho código puede alcanzar, algo equivalente a las reglas de formación proposicional.

Los trabajos experimentales a nivel de laboratorio que aportan la evidencia para sustentar los desarrollos teóricos aquí referidos han utilizado en su mayoría material verbal elaborado, controlado y manipulado con fines de investigación. El uso habitual del lenguaje en el marco de la interacción comunicativa supone, en cambio, casi siempre, la convivencia entre el código verbal y otros códigos de representación y comunicación, como ocurre con los llamados textos multimodales (Schnotz, Bannert \& Seufert, 2002; Schnotz \& Baadte, 2015; Schüler, Arndt \& Scheiter, 2015), el cine, el teatro, la publicidad audiovisual, los periódicos electrónicos y muchos otros objetos multisemióticos complejos de uso cotidiano. En este contexto cabe preguntarse qué propiedades constitutivas caracterizan la representación mental de estos mensajes producto de la comprensión de los mismos. ¿Se trata de un modelo de situación corporeizado, análogo? ¿Se acerca más a un modelo de inspiración proposicional? ¿Depende ello de la naturaleza del objeto multisemiótico y el predominio en él de un código por sobre otros? ¿Cuál es el papel del lenguaje en este proceso? Recientemente, Parodi y Julio (2015) demostraron que sí es posible, bajo determinadas condiciones, comprender un texto multisemiótico desde un solo código predominante. Sin duda, estos y otros hallazgos no hacen sino abrir ventanas a un fenómeno del que todavía se requiere mayor investigación para alcanzar propuestas teóricas formalizadas que integren en un solo modelo explicativo diversos códigos semióticos y sus correspondientes modelos mentales, como los intentos de Schnotz, Sadoski, Paivio y otros (Schnotz, Bannert \& Seufert, 2002; Sadoski \& Paivio, 2007; Sadoski, McTigue \& Paivio, 2012; Schnotz \& Baadte, 2015). 


\section{COMENTARIOS DE CIERRE}

Para finalizar, téngase en consideración que, si bien el lenguaje puede ser visto como el gran articulador de las representaciones mentales humanas, es necesario que se cumplan varios requisitos antes de que los niños empiecen a producir sus primeras palabras. Numerosas evidencias muestran que entre los 2 y 3 meses de vida el bebé humano puede percibir objetos individualizados y reconocerlos más tarde. Asimismo, existe una incipiente capacidad para percibir la figura, silueta, consistencia, superficie, color y otras propiedades perceptibles en forma visual y táctil de los objetos con los que los niños se relacionan. Sobre esta base, les resulta posible abstraer características de una entidad utilizando categorías para elaborar las representaciones (Baillargeon, 2004; Johnson, 2004; Poulin-Dubois \& Graham, 2007). En otras palabras, antes de hablar, los niños ya están formando rudimentarios, pero efectivos, modelos mentales. Más tarde, con la aparición del lenguaje, adquirirán las herramientas para formar expresiones lineales (significantes) que constituirán el soporte para transmitir comunicativamente dichos contenidos (significados), los que finalmente podrán ser comprendidos por su interlocutor solo reconstruyendo el modelo de situación correspondiente.

Como contraparte, el envejecimiento trae consigo profundos cambios en las capacidades cognitivas y lingüísticas del individuo (Véliz, Riffo \& Arancibia, 2010); sin embargo, a diferencia de la declinación observada en el procesamiento a nivel de representaciones de superficie y de base de texto, tal disminución no alcanza grados relevantes para efectos de la elaboración y actualización del modelo de situación (Radvansky, 1999; Radvansky \& Dijkstra, 2007), siendo este uno de los aspectos de mayor resistencia. Se refuerza así la idea de que en el complejo universo de la mente humana, el lenguaje convive con otras formas de representación evolutivamente anteriores. De los avances de la investigación dependerá que se logre explicar con mayor precisión el lugar que le corresponde a cada una de ellas y los mecanismos específicos que las relacionan. 


\section{REFERENCIAS BIBLIOGRÁFICAS}

Anderson, J. \& Bower, G. (1973). Human associative memory. Washington, D.C.: Winston.

Atkinson, R. \& Shiffrin, R. (1968). Human memory: A proposed system and its control processes. En K. Spence \& J. Spence (Eds.), The psychology y of learning and motivation (pp. 89-195). Nueva York: Academic Press.

Baddeley, A. (1986). Working memory. Nueva York: Oxford University Press.

Baddeley, A. (2000). The episodic buffer: A new component of working memory? Trends Cognitive Science, 4(11), 417-423.

Baillargeon, R. (2004). Infants' reasoning about hidden objects: Evidence for eventgeneral and event-specific expectations. Developmental Sciences, 7, 391-422.

Barsalou, L. (1999). Perceptual symbol systems. Behavioral \& Brain Sciences, 22, 577-660.

Belinchón, M., Igoa, J. \& Rivière, A. (2005). Psicología del lenguaje. Investigación y teoría. Madrid: Trotta.

de Vega, M. (2005). Lenguaje, corporeidad y cerebro: Una revisión crítica. Revista Signos. Estudios de Lingüística, 38(58), 157-176.

de Vega, M., Carreiras, M., Gutiérrez-Calvo, M. \& Alonso-Quecuty, M. (1990). Lectura y comprensión. Una perspectiva cognitiva. Madrid: Alianza.

de Vega, M., Glenberg. A. \& Graesser, A. (Eds.) (2008). Symbols and Embodiment. Debates on meaning and cognition. Oxford: Oxford University Press.

Frege, G. (1918). Logische Untersuchungen. Erster Teil: Der Gedanke. Beiträge zur Philosophie der deutschen Idealismus, 2, 58-77.

Fodor, J. A. (1983). The modularity of mind. Cambridge, MA: MIT Press.

Garrod, S. \& Sanford, A. J. (1983). Topic dependent effects in language processing. En G. Flores D'Arcais \& R. Jarvella (Eds.), The process of language understanding (pp. 271-296). Chichester: Wiley \& Sons.

Gernsabcher, M. (1985). Surface information loss in comprehension. Cognitive Psychology 17, 324-363.

Gernsabcher, M. (1990). Language comprehension as structure building. Hillsdale, N.J.: Lawrence Erlbaum Associates.

Gernsbacher, M. \& Kaschak, M. (2013). Text comprehension. En D. Reisberg (Ed.), The Oxford Handbook of Cognitive Psychology (pp. 462-474). Londres: Oxford University Press. 
Glenberg, A., Meyer, M. \& Lindem, K. (1987). Mental models contribute to foregrounding during text comprehension. Journal of Memory and Language, 26, 69-83.

Graesser, A. (1981). Prose comprehension beyond the word. Nueva York: Springer-Verlag.

Haberlandt, K. \& Graesser, A. (1985). Component processes in text comprehension and some of their interactions. Journal of Experimental Psychology: General, 114, 357-374.

Hart, J. \& Kraut, M. (2007). Neural basis of semantic memory. Cambridge: Cambridge University Press.

Johnson, S. (2004). Development of perceptual completion in infancy. Psychological Science, 15, 769-775.

Johnson-Laird, P. (1983). Mental models. Cambridge: Cambridge University Press.

Kintsch, W. (1974). The representation of meaning in memory. Hillsdale, N. J.: Lawrence Erlbaum.

Kintsch, W. (1988). The role of knowledge in discourse comprehension: A construction-integration model. Psychological Review, 95, 163-182.

Kintsch, W. (1993). Information accretion and reduction in text processing: Inferences. Discourse Processes, 16, 193-202.

Kintsch, W. (1998). Comprehension: A paradigm for cognition. Cambridge: Cambridge University Press.

Kintsch, W. \& Keenan, J. (1973). Reading rate and retention as a function of the number of propositions in the base structure of the sentences. Cognitive Psychology, 5, 257-274.

Kintsch, W., Welsch, D., Schmalhofer, F. \& Zimny, S. (1990). Sentence memory: A theoretical analysis. Journal of Memory and Language, 29, 133-159.

Langston, W., Kramer, D. \& Glenberg, A. (1998). The representation of space in mental models derived from text. Memory \& Cognition, 26, 247-262.

Liberman, A. \& Mattingly, I. (1985). The motor theory of speech perception revised. Cognition, 21, 1-36.

Marslen-Wilson, W. (1987). Functional parallelism in spoken word recognition. Cognition, 25, 71-102. 
McKoon, G. \& Ratcliff, R. (1992). Inference during reading. Psychological Review, 99, 440-466.

McNamara. D. \& Magliano, J. (2009). Toward a comprehensive model of comprehension. En B. Ross (Ed.), The Psychology of Learning and Motivation, Vol. 51 (pp. 297-384). Burlington: Academic Press.

Parodi, G. \& Julio, C. (2015). Más allá de las palabras: ¿Puede comprenderse el género discursivo Informe de Política Monetaria desde un único sistema semiótico dominante? Alpha, 41, 133-158.

Poulin-Dubois, D. \& Graham, S. (2007). Cognitive processes in early word learning. En E. Hoff \& M. Shatz (Eds.), Blackwell Handbook of Language Development (pp. 191-211). Malden, MA: Blackwell.

Pulvermüller, F. (2013). How neurons make meaning: Brain mechanisms for embodied and abstract-symbolic semantics. Trends in Cognitive Sciences, 17, 458470.

Radvansky, G. (1999). Aging, memory and comprehension. Current Directions in Psychological Science, 8, 49-53.

Radvansky, G. (2009). Spatial directions and situation model organization. Memory \& Cognition, 37, 796-806.

Radvansky, G. \& Dijkstra, K. (2007). Aging and situation model processing. Psychonomic Bulletin \& Review, 14, 1027-1042.

Ratcliff, R. (2012). Response time distribution. En H. Cooper (Ed.), APA Handbook of Research Methods in Psychology (pp. 429-458). Washington, DC: APA.

Ratcliff, R. \& McKoon, G. (1978). Priming in item recognition: Evidence for the propositional structure of sentences. Journal of Verbal Learning \& Verbal Behavior, 17, 403-417.

Rayner, K. \& Kiegl, R. (2012). Eye movements and cognitive processes. En H. Cooper (Ed.), APA Handbook of Research Methods in Psychology (pp. 413-427). Washington, DC: APA.

Rumelhart, D. E. (1980). Schema: The building blocks of cognition. En R. Spiro, B. Bruce \& W. Brewer (Eds.), Theoretical issues in reading comprehension (pp. 33-58). Hillsdale, NJ: LEA.

Sadoski, M. \& Paivio, A. (2007). Toward a unified theory of reading. Scientific Studies of Reading, 11, 337-356. 
Sadoski, M., McTigue, E. \& Paivio, A. (2012). A dual coding theoretical model of decoding in reading: Subsuming the leberge and Samuels Model. Reading Psychology, 33, 465-496.

Sanford, A. \& Garrod, S. (1981). Understanding written language: Explorations in comprehension beyond the sentence. Chichester: Wiley.

Saussure, F. (1916) [1945]. Curso de lingüística general. Buenos Aires: Losada.

Schüler, A., Arndt, J. \& Scheiter, K. (2015). Processing multimedia material: Does integration of text and pictures result in a single or two interconnected mental representations? Learning and Instruction, 35, 62-72.

Schnotz, W. \& Baadte, Ch. (2015). Surface and deep structures in graphics comprehension. Memory \& Cognition, 43, 605-618.

Schnotz, W., Bannert, M. \& Seufert, T. (2002). Toward an integrative view of text and picture comprehension: Visualization effects on the construction of mental models. En J. Otero, J. León \& A. Graesser (Eds.), The psychology of science text comprehension (pp. 385-416.). Mahwah, N.J.: Lawrence Erlbaum.

Speer, N., Reynolds, J., Swallow, K. \& Zacks, J. (2009). Reading stories activates neural representations of visual and motor experiences. Psychological Science, 20, 989-999.

Squire, L. (1987). Memory and brain. Nueva York: Oxford University Press.

Therriault, D., Rinck, M. \& Zwaan, R. (2006). Assessing the influence of dimensional focus during situation model construction. Memory \& Cognition, 34, 78-89.

Tulving, E. (1972). Episodic and semantic memory. En E. Tulving \& W. Donaldson (Eds.), Organization of Memory (pp. 382-402). Nueva York: Academic Press.

Van Dijk, T. \& Kintsch, W. (1983). Strategies of discourse comprehension. Nueva York: Academic Press.

Véliz, M., Riffo, B. \& Arancibia, B. (2010). Envejecimiento cognitivo y procesamiento del lenguaje: cuestiones relevantes. $R L A, 48,75-103$.

Vieiro, P. \& Gómez, I. (2004). Psicología de la lectura: Procesos, teorías y aplicaciones instruccionales. Madrid: Pearson.

Zwaan, R. (1996). Processing narrative time shifts. Journal of Experimental Psychology: Learning, Memory and Cognition, 22, 1196-1207.

Zwaan, R. (2003). The immersed experiencer: Toward an embodied theory of language comprehension. Psychology of Learning and Motivation, 44, 35-62. 
Zwaan, R. (2015). Situation models, mental simulations, and abstract concepts in discourse comprehension. Psychonomic Bulletin \& Review, 23, 1028-1034.

Zwaan, R., Langston, M. \& Graesser, A. (1995). The construction of situation models in narrative comprehension: An event-indexing model. Psychological Science, 6, 292-297.

Zwaan, R. \& Radvansky, G. (1998). Situation models in language comprehension and memory. Psychological Bulletin, 123, 162-185.

\section{NOTAS}

${ }^{1}$ El término 'psiquico' empleado por Saussure en el Curso de Lingüística General se refiere, a nuestro juicio, al carácter 'psicológico' del fenómeno, por lo que usaremos esta forma en adelante en el presente artículo.

2 Tal interpretación errónea ha tenido cierto eco en la enseñanza de la Lingüística otorgando solo al significado una naturaleza mental.

${ }^{3}$ Utilizaremos en este artículo los términos ‘texto’ y 'discurso' en forma indistinta.

${ }^{4}$ Una tercera opción, muchísimo menos estudiada, por cierto, es de naturaleza táctil, como la escritura en Braile, que no consideramos en el presente artículo.

${ }^{5}$ La psicología distingue, dentro de la memoria sensorial, dos modalidades llamadas memoria icónica y memoria ecoica, correspondientes a mecanismos implicados en la percepción visual y auditiva, respectivamente.

${ }^{6}$ Llamada también memoria operativa en gran parte de la literatura en lengua española.

${ }^{7}$ En sistemas de escritura no fonológica, el proceso de conversión de las unidades gráficas a unidades lingüísticas requiere operaciones un tanto distintas. No es el objetivo del presenta artículo profundizar en dichas diferencias. Además, la mayor parte de la investigación y, sobre todo los modelos teóricos, han centrado la atención en los procesos de comprensión de textos escritos en sistemas ortográficos de carácter fonológico.

${ }^{8}$ La lingüística del texto propuso la noción de superestructura para dar cuenta del esquema textual prototípico que caracteriza diversas formas identificables como relato, noticia, carta, aviso publicitario, ensayo, poema, etc. En la actualidad, la noción de 'género discursivo' ha surgido como alternativa que incluye aspectos estructurales, pero también los supera mediante la incorporación de la dimensión pragmática y otros elementos de índole cultural que hacen de dicha noción una propuesta teórica más compleja y explicativamente más poderosa. Con todo, no es el propósito de este artículo profundizar en ello ni menos optar por una de ambas nociones como alternativa. 
${ }^{9}$ Fregue otorgaba a las proposiciones una función operativa para efectos de razonamiento lógico completamente desprovisto de implicancias psicológicas o mentales. De hecho, el filósofo alemán las inventó como parte de un lenguaje formal y universal de inferencia lógica.

${ }^{10}$ Utilizaremos mayoritariamente estas tres expresiones en forma indistinta para referirnos a una forma de representación del discurso supralingüística y modal. En algunos puntos del artículo será necesario establecer distinciones específicas entre ellas en función de aclarar diferencias teóricas puntuales; de lo contrario, deben considerarse como la misma clase de objetos mentales. 